\title{
Los objetivos de desarrollo sostenible: una encrucijada paradigmática de la sociedad globalizada*
}

\author{
The sustainable development goals: \\ a paradigmatic crossroads of the globalized society
}

Os objetivos do desenvolvimento sustentável: uma encruzilhada paradigmática da sociedade globalizada

Fecha de entrega: 28 de octubre de 2015

Fecha de evaluación: 5 de mayo de 2016

Fecha de aprobación: 15 de julio de 2016

Javier Collado Ruano**

\section{Resumen}

El objeto de investigación del presente artículo es identificar y definir las diferentes perspectivas epistemológicas y metodológicas que han

* El artículo es el resultado de la investigación doctoral en la Universidad Federal de Bahia en Brasil que el autor se encuentra realizando actualmente. DOI: http://dx.doi.org/10.15332/s0120-8462.2016.0115.06

* $\quad$ Profesor e investigador de la Universidad Nacional de Educación (UNAE) de Ecuador. Doctor en Difusão do Conhecimento por la Universidad Federal de Bahía (Brasil). Doctor en Filosofía por la Universidad de Salamanca (España). Máster en Sociología de la Educación por la Universidad de Sevilla (España). Licenciado en Historia por la Universitat de València (España) con especialización en Relaciones Internacionales por la Universidad Degli Studi di Palermo (Italia). Trabajó como voluntario internacional en proyectos de cooperación al desarrollo en las áreas rurales de Perú y Ecuador. Presidente y fundador de la ONG Educar para Vivir (www.educarparavivir.com). Director de edición del e-journal Global Education Magazine, apoyado por la Unesco y UNHCR (www.globaleducationmagazine.com). Puede leer sus trabajos, entrevistas y publicaciones aqui: http://ufba.academia.edu/javiercolladoruano. Correo electrónico: javiercolladoruano@gmail.com 
emergido desde la mitad del siglo XX: la multidisciplinariedad, la pluridisciplinariedad, la interdisciplinariedad y la transdisciplinariedad. También aborda la irrupción de la globalización y los procesos transfronterizos haciendo un acercamiento genealógico a la epistemología del sur latinoamericana, africana, islámica y asiática. Por último, reivindica la necesidad de crear puentes transculturales entre los pueblos de todo el mundo para superar la encrucijada paradigmática en la que nos encontramos como sistema-mundo, con la finalidad de alcanzar los objetivos de desarrollo sostenible establecidos por las Naciones Unidas para el año 2030.

Palabras clave: globalización, epistemología del sur, estudios subalternos, paradigma otro, objetivos de desarrollo sostenible, transdisciplinariedad, pensamiento fronterizo, ecología de saberes, sistema-mundo, capitalismo.

\section{Abstract}

The research object of this article is to identify and define the different epistemological and methodological perspectives that have emerged since the mid-twentieth century: multidisciplinarity, pluridisciplinarity, interdisciplinarity and transdisciplinarity. It also addresses the irruption of globalization and cross-border processes making a genealogical approach to the epistemology of the South: Latin American, African, Islamic, and Asian. Finally, it claims the need for cross-cultural bridges between peoples worldwide to overcome the paradigmatic crossroads at which we find ourselves as a world-system, with the aim to achieve the Sustainable Development Goals set by the United Nations for 2030.

Keywords: Globalization, south epistemology, subaltern studies, another paradigm, Sustainable Development Goals, transdisciplinarity, border thinking, ecology of knowledge, world-system, capitalism. 


\section{Resumo}

O objeto de pesquisa de este artigo é identificar e definir as diferentes perspectivas epistemológicas e metodológicas que tem surgido desde a metade do século XX: a multidisciplinaridade, a pluridisciplinaridade, a interdisciplinaridade e a transdisciplinaridade. Também aborda a irrupção da globalização e os processos trans fronteiriços fazendo uma aproximação genealógica a epistemologia do sul latino-americano, africano, islâmico e asiático. Por último, reivindica a necessidade de criar pontes transculturais entre os povos do mundo todo para superar a encruzilhada paradigmática na qual nos encontramos como sistemamundo, com a finalidade de atingir os objetivos do desenvolvimento sustentável estabelecidos pelas Nações Unidas para o 2030

Palavras-chave: Globalização, epistemologia do sul, estudos subalternos, paradigma outro, objetivos de desenvolvimento sustentável, transdisciplinaridade, pensamento fronteiriço, ecologia de saberes, sistema-mundo, capitalismo.

\section{Iniciando...}

La era de la globalización está en continua evolución, como la vida en la Tierra o el propio universo. La sociedad red del siglo XXI sigue expandiéndose multidimensionalmente en diferentes niveles de realidad (local, regional, nacional e internacional): generando una extensa red de interdependencia universal de fenómenos políticos, económicos, tecnológicos, ecológicos, culturales, etc. (Castells, 2000). La competición económica caracterizada por el crecimiento irracional de las sociedades industriales ha puesto de manifiesto la insostenibilidad del sistema de producción capitalista para las futuras generaciones. La explotación de los recursos naturales de forma descontrolada es un tema de preocupación internacional donde diferentes actores geopolíticos -internacionales, regionales, nacionales y locales- investigan y analizan, desde hace décadas, los fenómenos transfronterizos que afectan en la vida de las personas. En la Cumbre del Milenio de las Naciones Unidas de New York del año 2000, se produjo un hito histórico importante de cooperación intergubernamental que podríamos 
comparar con la propia constitución de las Naciones Unidas en octubre de 1945, cuando la humanidad entera se encontraba amenazada de convertirse en una enorme atmósfera de "cenizas radioactivas". Los albores del tercer milenio comenzaron con el acuerdo de 189 países miembros para alcanzar ocho metas globales de desarrollo humano para el 2015: los objetivos de desarrollo del milenio (ODM). Un acuerdo histórico donde los estados soberanos reafirmaron los compromisos adquiridos en las conferencias precedentes de Estocolmo (1972), el trabajo de la "comisión Brundtland" con el informe Nuestro futuro común (1987), la Cumbre de la Tierra (1992), el Programa de Acción de Barbados (1994), la Cumbre sobre Desarrollo Social de Copenhague (1995), el protocolo de Kyoto (1997), así como otros encuentros de connotaciones destacables.

En septiembre de 2015 las Naciones Unidas han establecido un nuevo marco de acción para el 2030 que requieren un esfuerzo de cooperación internacional: objetivos de desarrollo sostenible (ODS). Los ODS demandan una organización del conocimiento con nuevas fórmulas políticas transfronterizas puesto que constituyen un reto de gobernabilidad mundial sin precedentes históricos que requieren desarrollar nuevas sinergias multidimensionales de carácter glocal $^{1}$ entre la ciudadanía planetaria actual y futura. No podemos mantener el orden socioeconómico capitalista actual, pues resulta incompatible con los límites del planeta Tierra. En palabras del filósofo moral Jorge Riechmann (2014): "no hay recursos naturales ni espacio ecológico suficiente para que la forma de producir y consumir hoy dominante en Estados Unidos, la Unión Europea o Japón se extienda al planeta entero" (p. 24). La crisis económica y ecológica global es en realidad una crisis de civilización planetaria. La ciudadanía global del siglo XXI necesita nuevas herramientas para comprender la realidad y herramientas para transformarla. Se requiere la formación, promoción e integración de una nueva consciencia humana que nos responsabilice con la situación de emergencia actual caracterizada por la insostenibilidad del sistema capitalista actual. Pero la creación de una auténtica educación libertadora que pretenda conseguir los ODS también implica una ruptura radical con las estructuras político-económicas y socio-educativas del pasado, ya que la educación tecnocrática, todavía vigente en la mayoría de los ámbitos formales, está basada en el modelo de organización social alienante que el capitalismo ha impuesto, desde la creación de los Estados modernos

1 El término glocal es acuñado por R. Robertson (1992) y es un neologismo formado por las palabras globalización y localización. Para Robertson (1992), la globalización no implica una anulación de lo local, sino una inclusión, presencia y encuentro de y con las culturas locales. 
y la Revolución Industrial, para reducir a los estudiantes a consumidores sumisos y a ciudadanos pasivos.

La insostenibilidad generada por la sociedad postindustrial actual ha puesto de manifiesto la necesidad de crear, difundir y gestionar el conocimiento a través de un nuevo abordaje epistemológico que cuestione el legado recibido de la ciencia positivista que ha sido hegemónica entre los siglos XVIII y XX. Si bien es cierto que gracias a estas escuelas occidentales de pensamiento de reducción epistemológica hemos obtenido un gran desarrollo tecnológico y material, la propia especialización disciplinar ha puesto en jaque las fronteras conceptuales y metodológicas del reduccionismo epistemológico en que la ciencia moderna se apoyaba. Esta situación ha permitido la emergencia de nuevos diálogos entre las propias disciplinas científicas. El conocimiento científico especializado (mono) disciplinar no puede tratar en solitario los problemas políticos, ecológicos y epistemológicos que los ODS demandan. Se necesita una ecología de saberes (Santos, 2010a) cuyo abordaje epistemológico abarque los saberes no científicos subyacentes en las culturas ancestrales, la sabiduría indígena, las artes y otras formas de organización del conocimiento que incluyan las inter-retro-acciones del género humano consigo mismo, con el otro, con la naturaleza y con la totalidad cósmica que sustenta lo sagrado. De ahí la importancia en identificar y definir las formas de colaboración disciplinar más destacadas.

\section{La colaboración más allá de las disciplinas para superar el paradigma de la simplificación}

Simbólicamente, el pasaje del siglo XIX al siglo XX fue denominado como la nueva Babel. El corpus de conocimiento científico adquirido en ese periodo dio lugar a una inflación del conocimiento y a la pulverización de la ciencia en una infinidad de disciplinas hiperespecializadas, la creación de nuevas dinámicas curriculares, así como la incorporación de nuevos modelos epistemológicos. Esta situación llevó a Thomas S. Kuhn (1970) y Paul Feyerabend (1997) a introducir la noción de inconmensurabilidad en el campo de filosofía de la ciencia, de manera independiente, en 1962. En este sentido, resulta elocuente la comunicación Notes toward a social epistemology of transdisciplinarity presentada por Julie Thompson Klein durante el I Congreso Mundial de la Transdisciplinariedad celebrado en Arrábida (Portugal) en 1994. En 
ella, Klein (1994) señalaba que de las 7 a 54 disciplinas identificadas entre 1939 y 1950, podrían constatarse 8.530 áreas del conocimiento definibles ${ }^{2}$ en el año 1987.

En este contexto de mitad del siglo XX la revolución cuántica tuvo un fuerte impacto cultural y científico que significó el cuestionamiento del dogma filosófico contemporáneo fundamentado en la existencia de un único nivel estructural de realidad ontológica. A diferencia del paradigma de la simplificación derivado de los postulados de la física clásica, la nueva lógica subyacente a la mecánica cuántica comenzó a observar el espacio entre las disciplinas con la misma potencialidad que al vacío cuántico. De acuerdo con los postulados de la mecánica cuántica, el vacío contiene ondas electromagnéticas fluctuantes y partículas elementales emergentes, cuya masa e interacciones posibilitan estados distintos y, por ende, vacíos diferentes. Según el físico teórico Nicolescu (2008) la relación entre las partículas y el vacío es similar a la relación en el vacío disciplinar. Desde un pensamiento clásico no existe nada entre las disciplinas, ningún tipo de energía-materia que las circunde para interconectarlas. Sin embargo, cuando observamos dicho vacío disciplinar desde un marco epistemológico transfronterizo y con metodologías dialogantes, se observan diferentes tipos de interrelaciones. La cooperación entre disciplinas representa una fuente de innovación que se manifiesta como una nueva frontera de pensamiento necesaria para reformar los problemas derivados de la reducción y de la fragmentación a la que fueron sometidas la ciencia, la realidad humana y la estructura de la naturaleza. Estas interrelaciones disciplinares suponen un nuevo diálogo epistemológico para la construcción y gestión del conocimiento que la sociedad globalizada actual está demandando para alcanzar los ODS en 2030. Siguiendo la definición axiomática de Nicolescu (2008) podemos sintetizarlas estas interrelaciones del siguiente modo:

2 La búsqueda de una explicación integrada a tales hechos, nos traslada al contexto de las grandes transformaciones politicas del periodo ulterior a la II Guerra Mundial, con la denominada Guerra Fría y la hegemonía económica de los Estados Unidos de América. La lógica adyacente en el capitalismo de la época exigía conocimientos cada vez más especializados para la producción en serie. 
Disciplinariedad: representa la forma de pensar la realidad conforme a las exigencias del método científico moderno, donde el conocimiento es fragmentado y convertido en objeto. El conocimiento se organiza en diversas disciplinas o áreas específicas, donde cada una de ellas posee sus propias reglas metodológicas, produciéndose una especialización atomizada. A su vez, las interrelaciones dentro de una disciplina, en función de su lógica interna, se definen como intradisciplinariedad.

Multidisciplinariedad: se ocupa de estudiar un tema de investigación desde varias disciplinas simultáneamente. Desde esta perspectiva, cualquier tema se verá enriquecido con la incorporación de los puntos de vista de varias disciplinas. Así pues, el enfoque multidisciplinario excede los propios límites disciplinarios, pero su objetivo sigue siendo limitado al marco de la investigación disciplinaria, ya que las disciplinas cooperan en forma mutua y acumulativa, pero no interactiva. Los profesionales implicados en una tarea multidisciplinar adoptan relaciones de colaboración con objetivos comunes.

\section{Fragmentación}

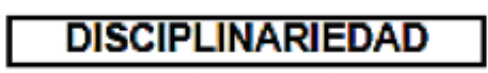

Organización por áreas del conocimiento

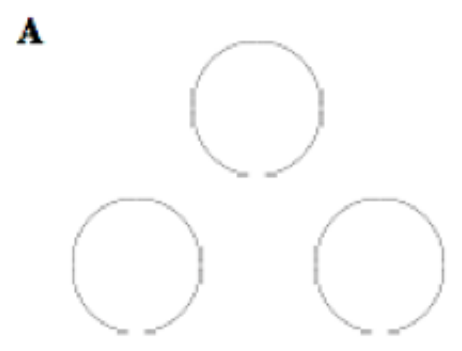

Sin diálogo

- Cada área con metodología propia

- Especialización atomizada

Figura 1. Disciplinariedad. Elaboración propia.

\section{Genérica}

(Interdependecia agrupada)

\section{MULTIDISCIPLINARIEDAD \\ Coordinación por estandarización \\ Inversión en todo el sistema}

B

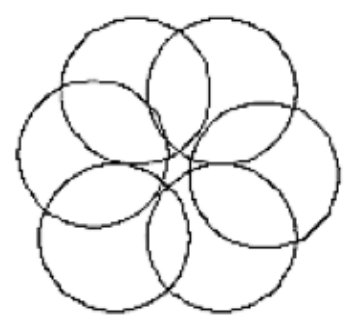

Introducir información

- Cada disciplina representada

- Múltiples sistemas cerrados interactúan con un sistema abierto

Figura 2. Multidisciplinariedad. Elaboración propia. 
Pluridisciplinariedad: estudio de un objeto de una misma y única disciplina por varias disciplinas, situadas generalmente en el mismo nivel jerárquico, al mismo tiempo. El abordaje pluridisciplinar sobrepasa las disciplinas a través de una interacción o cooperación disciplinar, donde los métodos propios de cada una son conservados, y cuya finalidad continúa inscrita en la estructura de investigación disciplinar.

\section{Interacción}

\section{PLURIDISCIPLINARIEDAD \\ Cooperación disciplinar en un mismo nivel jerárquico}

G
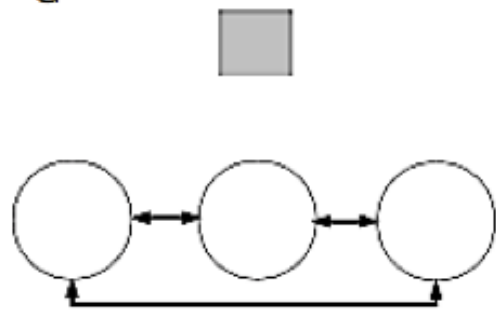

Ultrapasa las disciplinas

- Métodos propios conservados

- Estudio de un objeto disciplinar por varias disciplinas

Figura 3. Pluridisciplinariedad. Elaboración propia.

Interdependencia secuencial

Interdisciplinariedad: interacción prolongada y coordinada entre disciplinas académicas, llevando a la integración de los diferentes discursos y a la creación de un léxico o cuadro conceptual común, formando puentes entre las grietas de las estructuras disciplinares, llegando a formular una metodología común, transcendiendo la interface de las epistemologías de diferentes disciplinas. La interdisciplinariedad se organiza en dos niveles jerárquicos, puesto que se introduce un sentido de propósito cuando la axiomática común a un grupo de disciplinas se define en el nivel jerárquico inmediatamente superior. Se pueden distinguir tres tipos de grados: a) de aplicación; b) epistemológico; c) de generación de nuevas disciplinas (ejemplo: teoría del caos, cosmología cuántica).

\section{INTERDISCIPLINARIEDAD}

Coordinación por planificación Objetivos intersistémicos

D

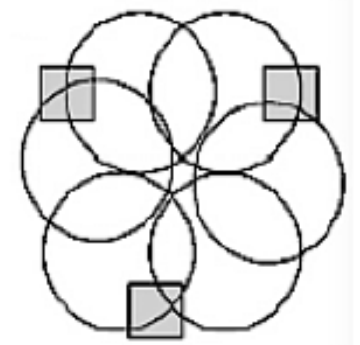

\section{Rendimiento}

- Cada disciplina contribuyendo

- Interacción disciplinar planeada

Figura 4. Interdisciplinariedad. Elaboración propia. 
Transdisciplinariedad: desarrollo de una axiomática general cruzando la esencia de las disciplinas. Interacción prolongada y coordinada entre disciplinas académicas y conocimientos producidos por los sujetos fuera de la academia, en un proceso de aprendizaje recíproco y sin jerarquía, para la resolución de determinados problemas complejos que no pueden ser resueltos por abordajes monodisciplinares. Esa interacción puede llevar a la integración de los diferentes discursos de las disciplinas y de los conocimientos no académicos (pueblos originarios, artísticos, musicales, etc.), mediante la creación de un lenguaje o de un cuadro conceptual común, llegando a formular una metodología común, transcendiendo la interface de epistemologías de los diferentes conocimientos académicos y no académicos y generando un nuevo tipo de conocimiento. Compete al metapunto de encuentro entre las disciplinas y a la concepción más allá de las disciplinas. Su objetivo es la comprensión ética del mundo presente y la promoción de una cultura de paz, para el cual uno de los imperativos es la unidad del conocimiento.
Interdependencia reciproca

\section{TRANSDISCIPLINARIEDAD \\ Coordinación basada en objetivos individuales y sistérnicos en diélogo}

Inversión en incertidumbre por la interdependencia combinada y secuencial

$\mathbf{E}$

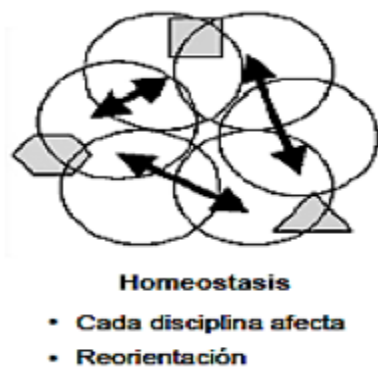

Figura 5. Transdisciplinariedad. Elaboración propia.

Como vemos, en las últimas décadas el conocimiento viene organizándose a través de nuevos acercamientos metodológicos que nos permiten aproximarnos a la compleja red de fenómenos sociales y naturales que constituyen el sistema-mundo. Gracias a estos nuevos abordajes metodológicos podemos discurrir que la cosmovisión paradigmática que tenemos del sistema-mundo son interpretaciones de la realidad en que vivimos, las cuales reflejan, a su vez, el cuadro epistemológico que engloba los paradigmas sociales existentes. Es decir, el modo introspectivo en que experimentamos y comprendemos los procesos que condicionan las formas de entendimiento 
e interpretación del mundo, los cuales están en el núcleo mismo de las creencias paradigmáticas de una determinada época histórica. Así, la hegemonía de un cierto tipo de lectura de la realidad (o de los niveles de realidad), está impregnada en nuestro ser por el hecho de encontrarnos circunscritos a un paradigma concreto que actúa como referencial epistémico-cultural de nuestro mundo interior. Esta reflexión conceptual de paradigma es fundamental para comprender los procesos de control simultáneo en la relaciones lógicas y semánticas de un determinado discurso que privilegia cierto tipos de relaciones en detrimento de otras (Morin, 2008). Es lo que ocurre con el discurso cultural actual impuesto por el occidente capitalista al afirmar que todo crecimiento económico es bueno por sí mismo. En realidad, postular que los niveles de calidad humana se miden por el PIB y el PNB de un país supone cometer un fraude intelectual de peligrosas consecuencias en la era de la crisis ecológica global (Riechmann, 2014). Si bien es cierto que el sistema capitalista ha traído enormes beneficios materiales, su visión funcionalista subordina todo al máximo beneficio económico y al consumo indiscriminado en detrimento de la naturaleza. Todo lo que consumimos proviene de la regeneración biofísica de esta, que ya no da cuenta de los recursos demandados para mantener esta ilusión epistémica de crecimiento. Debemos salir de estas dinámicas fatalistas que nos conducen a la barbarie, y para ello tenemos que reformular paradigmáticamente la relación colonial que los humanos ejercemos sobre la naturaleza, pero también sobre nosotros mismos, ya que el pensamiento de frontera alberga la estrategia de que las clases marginales desafíen a las clases poderosas para lograr un mundo cosmopolita orientado hacia la justicia social y la equidad.

\section{"Un paradigma otro": una perspectiva epistémica del sur}

En las últimas décadas viene perfilándose un nuevo tipo de reflexión epistemológica descolonial que se sitúa del lado de la ciudadanía mundial del sur. Esta corriente de pensamiento cuestiona la dominación epistemológica occidental que eliminó y descontextualizó, durante siglos, el saber de los pueblos y de las naciones colonizadas. Se trata de una visión epistemológica conocida como epistemología del sur que se caracteriza por albergar un diálogo horizontal con los conocimientos de los subalternos colonizados en una ecología de saberes. Entre los pensadores más destacados que reflexionan a partir del sur encontramos a Enrique Dussel, Immanuel Wallerstein, Milton Santos, Ebrahim Moosa, Aníbal Quijano, Walter Mignolo, Boaventura de Sousa Santos, Ramón Grosfoguel, Nelson Maldonado-Torres, Mogobe B. Ramose, 
Amima Mama, Paulin Hountondiji, Rinajit Guha, Gayatri C. Spivak, Edward Said, Raewyn Connell, Dipesh Chakrabarty, Partha Chatterjee y otros muchos. La complejidad de este movimiento es difícil de entender sin reconocer el esfuerzo colectivo e interdependiente que existe en el desarrollo de una epistemología del sur crítica con la historiografía que narra la modernidad y la postmodernidad desde posiciones eurocéntricas/occidentales. Se trata de un movimiento intelectual donde convergen diferentes líneas de investigación y percepción epistemológica entre pensadores que reivindican la presencia del sur global para transgredir el paradigma de imposición epistémica occidental de la sociedad globalizada actual. Una epistemología del sur que, en definitiva, "se asienta en tres orientaciones: aprender que existe el sur; aprender a ir para el sur; aprender a partir del sur y con el sur” (Santos, 1995, p. 508).

En un intento de originar distintos principios políticos, éticos, económicos y epistémicos de la civilización neoliberal actual, Walter Mignolo (2003) propone el concepto de "paradigma otro" en su obra Historias locales/diseños globales: colonialidad, conocimientos subalternos y pensamiento fronterizo. En ella, Mignolo (2003) llama "paradigma otro a la diversidad de formas críticas de pensamiento analítico y de proyectos futuros asentados sobre las historias y experiencias marcadas por la colonialidad, dominantes hasta ahora, asentadas sobre las historias y experiencias de la modernidad" (p. 20). Este es "el conector" entre quienes han experimentado el trauma, la ignorancia, la falta de respeto y la imposición del "progreso" como un valor del bienestar. En palabras del propio filósofo, semiótico y especialista en estudios poscoloniales subalternos, Walter Mignolo (2003):

Un "paradigma otro" es en última instancia el nombre que conecta formas críticas de pensamiento "emergentes" (como en la economía) en las Américas (latinos; afroamericanos; americanos nativos; pensamiento crítico en América Latina y el Caribe), en el norte de África, en el África subsahariana, en el sur de India y en el sur de Europa, y cuya emergencia fue generada por el elemento común en toda esta diversidad: la expansión imperial/colonial desde el siglo XVI hasta hoy (p. 20).

De este modo, el "paradigma otro" tiene implícita la multirreferencialidad y la diversalidad en la unidad del conocimiento transdisciplinar, puesto que no tiene un autor de referencia ni un origen común. Se trata de un paradigma epistemológico que está en armonía con el concepto de rizoma epistemológico creado por Gilles Deleuze y 
Félix Guattari (2006) en su obra Capitalismo y esquizofrenia, puesto que se trata de una corriente de pensamiento que aprehende las multiplicidades. Es decir, la genealogía de esta corriente epistemológica del sur se bifurca como el rizoma botánico entre la multiplicidad de historias locales y experiencias coloniales transformadas en crítica epistémica en diferentes espacios y momentos temporales. Asimismo, Mignolo (2003) continúa señalando que:

El "paradigma otro" es, en última instancia, el pensamiento crítico y utopístico que se articula en todos aquellos lugares en los cuales la expansión imperial/ colonial le negó la posibilidad de razón, de pensamiento y de pensar el futuro. Es "paradigma otro" en última instancia porque ya no puede reducirse a un "paradigma maestro", a un "paradigma nuevo" que se autopresente como la "nueva" verdad (p. 20).

Esta visión mignoliana significa que el "paradigma otro" no es coherente con el pensamiento epistemológico creado por la modernidad, pero tampoco con las críticas postmodernas eurocéntricas/occidentales que la globalización neoliberal tiene circunscrita en el raciocinio de científicos y filósofos, como sucede en los casos de Michael Foucault y Jacques Derrida, por ejemplo. La diferencia radica en que los movimientos intelectuales que critican la modernidad desde posicionamientos eurocéntricos/occidentales defienden "otro paradigma" postmoderno, uno "nuevo"; mientras que el grupo de pensadores que adoptan la perspectiva epistemológica de descolonización abogan por "un paradigma otro". La "otredad" del paradigma que Mignolo plantea lleva implícita la negación de la "novedad" y de la "universalidad abstracta" del proyecto moderno que continúa colonizando de forma invisible las mentes de los individuos.

De ahí que Mignolo (2003) señale que "la hegemonía de "un paradigma otro" será, utopísticamente, la hegemonía de la diversidad, esto es, "de la diversidad como proyecto universal" y no ya un "nuevo proyecto abstracto" (p. 20). Es por esto que el autor enfatiza que su libro no es la "presentación" del paradigma otro, sino una "contribución" a él. Según la visión mignoliana, el "paradigma otro" está formado por proyectos que tienen en común la perspectiva y la crítica a la modernidad desde la colonialidad, es decir, por proyectos que surgen de la toma de consciencia de que no se trata de "diferencias culturales", sino de "diferencias coloniales" que ocultan la colonialidad del poder. Esta toma de consciencia origina un "pensamiento fronterizo" 
cuya perspectiva de descolonización suscita al diálogo entre los movimientos sociales y los intelectuales para superar el dominio de la política cultural colonial. Un buen ejemplo es el Foro Social Mundial llevado a cabo anualmente por un movimiento heterogéneo transfronterizo que aboga por una alterglobalización diferente. Este pensamiento fronterizo emergente permite recartografiar las culturas académicas y no académicas, uniendo y borrando las fronteras entre el conocer sobre y conocer desde, ayudando a imaginar un mundo sin fronteras rígidas (nacionales o civilizacionales) donde el conocimiento surge con la propia vida, y no con los griegos. En última instancia, la globalización representa una paradoja al establecer y demoler fronteras simultáneamente, de ahí que las viejas historias locales estén emergiendo de nuevo para proyectarse en el futuro, lo que significa la diversidad como proyecto universal (Mignolo, 2003, p. 390). Algunos ejemplos de historias locales emergentes que constituyen "un paradigma otro" son los movimientos indígenas en América Latina como el protagonizado por la guatemalteca Rigoberta Menchú ${ }^{3}$ o el levantamiento del Ejército Zapatista de Liberación Nacional de 1994 en México, cuyos paradigmas epistémicos hacen una disrupción con las directrices racionales occidentales que pretenden ordenar y controlar el cambio social.

Desde una perspectiva similar Boaventura de Sousa Santos (1995, 2010a, 2010b) defiende una transición paradigmática que origine un "nuevo sentido común", ya que el paradigma cultural y epistemológico que se viene imponiendo globalmente como paradigma moderno y occidental es una versión construida en base a las necesidades de dominación colonial. La colonización es la otra cara de la moneda de la modernidad. Las experiencias culturales y epistemológicas que no estaban alienadas con el padrón moderno colonizador y capitalista eran reducidas, marginadas y relegadas a un segundo plano. De ahí que Santos (2010b) defienda un occidente "no occidentalista", concordando con Goody (2006) al pensar que una verdadera "historia global" solo podrá ocasionarse en el momento en que se superen transdisciplinarmente las perspectivas eurocéntricas, antieurocéntricas, occidentales y orientales. Para Santos (2010a, 2010b) es posible combatir estas perspectivas a partir de una iniciativa interepistemológica asentada en una ecología de saberes y en las relaciones interculturales entre tradiciones. Esta concepción epistemológica alberga una paradoja, ya que la diversidad de experiencias

3 Rigoberta Menchú es una indígena maya guatemalteca que fue galardonada con el Premio Nobel de la Paz en 1992, en reconocimiento a su lucha por la justicia social y la reconciliación etnocultural basada en el respeto a los derechos de los indígenas. 
humanas representa una pluralidad infinita de saberes epistemológicos en un mundo finito. En palabras del propio sociólogo del derecho portugués:

Siendo infinita, la pluralidad de saberes existentes en el mundo es intangible en cuanto tal, ya que cada saber solo da cuenta de ella parcialmente, a partir de su específica perspectiva. Pero, por otro lado, como cada saber solo existe en esa pluralidad infinita de saberes, ninguno de ellos se puede comprender a sí mismo sin referirse a los otros saberes. El saber solo existe como pluralidad de saberes tal como la ignorancia solo existe como pluralidad de ignorancias. Las posibilidades y los límites de comprensión y de acción de cada saber solo pueden ser conocidas en la medida en que cada saber se propone una comparación con otros saberes. Esa comparación es siempre una versión contraída de la diversidad epistemológica del mundo, ya que esta es infinita. Es, pues, una comparación limitada, pero es también un modo de presionar al extremo los límites y, de algún modo, de sobrepasarlos y deslocarlos. En esa comparación consiste lo que designo por ecología de saberes (Santos, 2010b, p. 543).

Aquí concuerdo con Santos en que existe un número ilimitado de ecologías de saberes, tan infinito como la propia diversidad epistemológica y cultural que alberga la propia ciudadanía mundial, donde la inconmensurabilidad y la incompletitud del conocimiento -recordando los teoremas de Gödel-implica que los individuos hagamos una selección hermenéutica con los saberes paradigmáticos que interactúan en nuestro contexto dado. Esta dificultad es reconocida por el propio Santos (2010b) al expresar que la propuesta conceptual de ecología de saberes confronta dos problemas: “a) cómo comparar saberes dada la diferencia epistemológica; b) cómo crear un conjunto de saberes que participa de un ejercicio dado de ecología de saberes ya que la pluralidad de saberes es infinita" (Santos, 2010b, p. 544). Para el primer problema de comparar saberes epistémicos diversos, Santos (2010b) propone la traducción recíproca entre "señales, símbolos, conjeturas, enigmas, pistas, preguntas, paradojas, ambigüedades, etc". (p. 545). Para confrontar el segundo problema, Santos (2010b) aboga por la artesanía de las prácticas, es decir, un descentramiento de los saberes que promocione prácticas sociales eficaces y libertadoras a partir de una interpelación cruzada de los límites y de las posibilidades de cada uno de los saberes en presencia. Por ejemplo, "la preocupación de la preservación de la biodiversidad puede llevar a una ecología entre el saber científico y el saber camponés o indígena" (Santos, 2010b, p. 546). Pero en ambos problemas, Santos (2010b) denota una asimetría compleja entre saberes 
epistemológicos que se manifiestan en mayor medida como asimetrías políticas. $\mathrm{Al}$ maximizar esta asimetría se incrementa la ignorancia respecto a otros saberes, llegando a declarar su inexistencia: "a este modo lo llamo fascismo epistemológico porque constituye una relación violenta de destrucción o supresión de otros saberes" (Santos, 2010b, p. 544). En otras palabras, la supresión forzosa de conocimientos indígenas y ancestrales llevada a cabo por la colonización europea, que todavía continúa hoy en día con nuevas formas neoliberales, es una forma de "epistemicidio" que empobrece al ser humano en su totalidad. Por el contrario, al reconocerse esta asimetría entre los propios saberes, se minimiza la diferencia epistemológica a través de comparaciones y traducciones interculturales que posibilitan el entendimiento horizontal. De ahí que Santos (2010a, 2010b) proponga valorizar la diversidad de los saberes para que la intencionalidad y la inteligibilidad de las prácticas sociales sea lo más amplia y democrática posible. Esta ecología de saberes es, en definitiva, una opción epistemológica y política contrapuesta al fascismo epistemológico impuesto por la expansión colonial europea.

Desde una perspectiva similar a Santos, el sociólogo y teórico político peruano Aníbal Quijano desarrolla el concepto de colonialidad a partir de un análisis de la situación latinoamericana. Influenciado por el pensamiento de Marx y Gramsci, Quijano $(2001,2010)$ hace una interpretación epistémica de la situación de dominación presente en el sur global. Para este autor la destrucción de la colonialidad del poder es uno de los factores determinantes de la lucha contra el padrón universal del capitalismo eurocéntrico/moderno. "La "racionalización" de las relaciones de poder entre las nuevas identidades sociales y geoculturales fue el sustento y la referencia legitimadora fundamental del carácter eurocéntrico del padrón de poder, material e intersubjetivo" (Quijano, 2010, pp. 119-120). Por esta razón, el pensador peruano cuestiona la naturalización de las experiencias, las identidades, las relaciones históricas de la colonialidad y la distribución geocultural del poder capitalista mundial, estableciendo un esquema para el estudio de estas implicaciones paradigmáticas: 1) colonialidad de la clasificación social universal del mundo capitalista;2) colonialidad de la articulación política y geocultural;3) colonialidad de la distribución mundial del trabajo; 4) colonialidad de las relaciones de género; 5) colonialidad de las relaciones culturales o intersubjetivas; y 6) dominación/explotación, colonialidad y corporeidad.

En este análisis diferencial de colonialidad también destaca la propuesta radical de geopolítica descolonial propuesta por el especialista en estudios étnicos Nelson 
Maldonado-Torres (2010), quien realiza un análisis crítico de varios filósofos europeos contemporáneos para manifestar que la filosofía moderna occidental convirtió a Europa en el centro epistémico del mundo. De un modo complementario, Ramón Grosfoguel (2006) propone ampliar el debate epistémico sobre descolonialidad y capitalismo global desde una perspectiva crítica del nacionalismo, del colonialismo y del fundamentalismo (sea este eurocéntrico o del llamado tercer mundo). La crítica epistemológica que Grosfoguel (2006) realiza sobre los estudios dedicados a la globalización, a los paradigmas de la economía política y a los análisis del sistema-mundo, es que estos no logran desprenderse de la matriz epistémica del poder: "el postmodernismo y el postestructuralismo como proyectos epistemológicos están atrapados en el canon occidental reproduciendo en sus esferas de pensamiento y de práctica una forma particular de colonialidad del poder y el conocimiento" (Grosfoguel, 2006, p. 21). Para salir del paradigma epistémico eurocéntrico/occidental moderno, colonial, capitalista y patriarcal, Grosfonguel (2006) apunta experiencias alternativas construidas a partir de un pensamiento de frontera cuyos proyectos utópicos tienen como objetivo la emancipación de las relaciones de poder en el sistema-mundo. Preguntándose, “ ¿cómo se ve el sistema-mundo si movemos el locus de enunciación del hombre europeo a una mujer indígena en América, a, digamos, Rigoberta Menchú, en Guatemala, o a Domitila, en Bolivia?" (p. 24), Grosfoguel (2006) consigue desplazar el lugar desde el cual están pensados los paradigmas. Con ello Grosfoguel (2006) también consigue poner en tela de juicio la perspectiva económica reduccionista del sistema-mundo, ya que la colonización de América a finales del siglo XV ocasionó, de manera simultánea, una compleja jerarquía epistémica paradigmática impuesta por el "hombre europeo, capitalista, militar, cristiano, patriarcal, blanco, heterosexual" (Grosfoguel, 2006, p. 25). Sin duda, el planteamiento epistémico desde una perspectiva subalterna racial y étnica consigue transgredir y descolonizar los paradigmas epistémicos tradicionales donde la economía política conceptualiza el capitalismo como un sistema global o mundial.

Los procesos y las dinámicas neoliberales que la globalización viene desarrollando han creado una lógica epistémica paradigmática donde se prima el beneficio económico del mercado frente a la dignidad, la seguridad e incluso la misma supervivencia del ser humano. Existe la sensación de que la vida ha dejado de constituir el valor central del proyecto civilizatorio humano en la Tierra. El mundo sagrado de los pueblos originarios, donde la naturaleza es venerada por su carácter mágico, se ha visto remplazado por paraísos fiscales que satisfacen la avaricia y la codicia 
mercantil humana. Ante esta peligrosa situación que se evidencia particularmente en el sur global -a excepción de Australia y Nueva Zelanda-, el filósofo especialista en ciencias políticas y relaciones internacionales Mogobe B. Ramose (2010) analiza la globalización a partir del concepto ubuntu, preguntándose, “¿puede la filopráxis ubuntu ser una de las respuestas al fundamentalismo económico contemporáneo en forma de globalización?” (p. 179).

Ubuntu es uno de los conceptos filosóficos, ontológicos y éticos africanos que actúa como principio organizador esencial de los pueblos que hablan lenguas bantúes, los cuales permanecen abiertos a cooperar con todos los seres humanos del mundo que buscan sustituir el fundamentalismo económico imperante en la globalización por la preservación de la vida. Como es sabido, las lenguas bantúes constituyen una subfamilia de lenguas Níger-Congo que son habladas en Angola, Botsuana, Camerún, Gabón, Kenia, Malaui, Mozambique, Namibia, República del Congo, República Democrática del Congo, Sudáfrica, Tanzania, Uganda, Zambia y Zimbabue. Para Ramose (2010) "la globalización puede ser cultural, religiosa, política y económica, [de ahí que sea] posible describir tal situación como una condición de dominación epistemológica empeñada en suprimir la búsqueda de reconocimiento mutuo y de paridad” (p. 181). Las raíces de la globalización contemporánea están profundamente arraigadas a la Revolución Industrial, particularmente en el Reino Unido, y con la subsecuente difusión e imposición global de este modelo económico a través de la colonización durante los viajes de "descubrimiento". A pesar de la descolonización y la "reconquista" de la soberanía de los Estados-Nación (casi siempre con largas guerras de independencia), las redes económicas de dominación han permanecido en el sur global por el carácter epistémico paradigmático que se sustenta en la búsqueda incansable de mano de obra barata en la "periferia" del mundo. En palabras de Ramose (2010):

El dislocamiento de la industria, la desregulamentación, las redes y la obtención del máximo lucro a cualquier costo constituyen el doma de la religión del fundamentalismo económico. Las sacerdotisas y los sacerdotes de esta religión predican apenas el evangelio y veneran apenas un dios, principalmente, la lucratividad del mercado. Para ellos, el mercado es el poder financiero que sustenta la contradicción del espacio, del tiempo y de la política, sin importarles las posibles consecuencias humanas y ambientales. La obtención de lucros sin restricción es su principal meta. Bendecidos, por tanto, son los creadores del 
lucro infinito, pues ellos substituyeron la ilusión del cielo eterno por el lucro infinito del mercado (pp. 183-184).

A través de la identificación de alguna de las líneas principales de dominación económica, política, cultural y epistemológica, Ramose (2010) cuestiona la religión del fundamentalismo económico presentando la alternativa africana ubuntu como una propuesta epistemológica que inspira otra forma de ser y estar en el mundo: "ubuntu indica, por tanto, una acción particular ya realizada, una acción o estado duradero de ser y una posibilidad para otra acción o estado de ser" (Ramose, 2010, p. 211). De este modo, Ramose apunta que la filosofía africana ubuntu reconoce que los procesos de intercambio perpetuo de flujos de energía, adyacente en las fuerzas de la vida en la naturaleza, no pertenecen a nadie. Haciendo alusión a dos tesis encontradas en la mayoría de lenguas africanas, Motho ke motho ka batho y Feta kgomo o tshware motho, Ramose procura problematizar con la organización social y económica mundial, contribuyendo a establecer una nueva perspectiva epistémica paradigmática para el debate internacional de los derechos humanos.

El significado esencial del primer aforismo postula que "ser humano es afirmar la humanidad propia a través del reconocimiento de la humanidad de los otros y, sobre tal embasamiento, establecer relaciones humanas respetuosas para con ellos" (Ramose, 2010, p. 212). Este principio de reconocimiento mutuo significa que el ser humano individual es un sujeto (y no un objeto) de valor intrínseco en sí mismo, en la medida en que reconoce la dignidad del otro. Despreciar al otro significa despreciarse a sí mismo. Los individuos somos entidades inacabadas que solo revelamos nuestras potencialidades y habilidades a través de las relaciones humanas. En la línea de este argumento, Ramose (2010) señala que "la filosofía africana indígena de los derechos humanos avanza a partir de la dignidad del ser humano y de la negación del absolutismo y del dogmatismo" (p. 212). Por otro lado, el segundo aforismo significa que cuando una persona se encuentra en la disyuntiva de escoger entre la riqueza y la preservación de la vida de otro ser humano, debe optar por la preservación de la vida. De acuerdo con esta premisa filosófica, el ser humano individual debe ser considerado como el valor básico y principal de todos los valores, ya que la organización social y política basada en la riqueza es la fuente de conflictos y guerras. En este aforismo africano existe una gran diferencia conceptual con la filosofía occidental de los derechos humanos, ya que, si bien es cierto que la concepción occidental también parte del principio de que el ser humano individual es el principal criterio de valor, 
hace mayor énfasis en "la idea del ser humano como una entidad fragmentada sobre la cual los derechos pueden ser agregados en la base de contingencia", a diferencia de la concepción africana que "subraya la idea del ser humano como una totalidad, teniendo sus derechos asegurados como tal" (Ramose, 2010, p. 213).

La toma de consciencia entre las diferencias epistémicas de la filosofía occidental y la filosofía africana deben guiarnos para la superación paradigmática que la globalización capitalista actual impone al resto del mundo, haciendo prevalecer la opción de preservar la vida en la Tierra ante las lógicas de enriquecimiento materialista impuestas por una minoría. Debemos hacer autocrítica del metasistema actual guiado por la irracionalidad económica de la globalización y debemos abordar la política internacional a través de una nueva concepción pluralista y polilógica de la ética, que inspire nuevos rumbos de navegación por las aguas del siglo XXI. Para ello necesitamos reformular la ética humana y realizar un esfuerzo metacognitivo intelectual, moral y afectivo que advierta la complejidad de los ODS desde la perspectiva filosófica ubuntu, cuyo polimorfismo etológico representa la sabiduría de aprender a crecer juntos como sociedad globalizada. Por este motivo, resulta imprescindible que todos los actores socioeducativos promovamos la filosofía africana ubuntu como metaestructura de pensamiento, sensibilización y entendimiento de la convergencia evolutiva sociobiológica y antropoética, puesto que la simbiosofía pluricultural ubuntu constituye un elemento emergente para la gestión ética de nuestro futuro común como especie en armonía con las otras formas de vida en la naturaleza.

Una lectura similar es realizada por Dismas A. Masolo (2010) desde la ética aplicada y la filosofía política y social, valorizando el conocimiento indígena africano desde formas epistemológicas que evitan las categorías coloniales oposicionales del tradicional y del moderno. En este sentido, Paulin Hountondji (2010) también identifica dos perspectivas sobre los estudios africanos -el conocimiento de África y los conocimientos africanos-, y con ello abre camino para una discusión sobre la naturalización del conocimiento en cuanto símbolo de persistencia de la relación colonial. Hountondji (2010) defiende que otra producción del conocimiento deberá producirse "a la par de una reapropiación crítica de los propios conocimientos endógenos de África y, más que eso, con una apropiación crítica del propio proceso de producción y capitalización del conocimiento" (p. 141). Esta actitud crítica también aparece en el pensamiento del teólogo Ebrahim Moosa (2010), que hace una lectura de la dicotomía entre el islam tradicional y el islam progresista a partir su experiencia en la comunidad musulmana minoritaria en la Sudáfrica del apartheid (segregación racial). 
Todas esas perspectivas epistemológicas de descolonización también se han desarrollado ampliamente en la India. La obra Elementary aspects of peasant insurgency in colonial India, del historiador hindú Ranajit Guha es considerada un clásico en el sur asiático por su influencia en los estudios subalternos de la década de 1980. Repensando el término subalterno definido por el italiano marxista Antonio Gramsci, que se refiere a aquellas personas o grupos de rango y posición inferior, bien sea por causa racial, de clase, género, orientación sexual, etnicidad o religión; el grupo de trabajo South Asian subaltern studies, formado por Guha y sus discípulos, hacen una crítica a la narrativa tradicional marxista sobre la historia de la India. Desde la perspectiva de estudios subalternos, la preocupación por la política y el poder abre un proceso de "deconstrucción" epistémica paradigmática en la historiografía, donde se busca reescribir la historia de la India a partir de la lucha entre el subalterno y la élite, es decir, entre las masas indígenas y la soberanía británica. En palabras del propio Guha (1982):

\begin{abstract}
La historiografía del nacionalismo indio ha sido dominada por largo tiempo por un elitismo -elitismo tanto colonialista como de la burguesía nacionalista- que compartía el prejuicio de que la construcción de la nación india y el desarrollo de su conciencia -su nacionalismo- que confirmaba este proceso, eran logros que pertenecían exclusiva o predominantemente a una élite. En las historiografías colonialistas y neocolonialistas estos logros se atribuían a la dominación británica, a los administradores coloniales, a sus agentes de control policial, a sus instituciones y a su cultura. En los escritos nacionalistas y neonacionalistas se atribuirían ahora a las personalidades, a las instituciones, a las actividades y a las ideas de una élite india (p. 1) (traducción propia).
\end{abstract}

De este modo, Guha muestra que la alternativa subalterna es un conocimiento integral para superar todas las lagunas, lapsos e ignorancias que el nacionalismo indiano alberga en su narración tradicional. Su afirmación es que la perspectiva del ser subalterno puede comprender toda la experiencia de la resistencia indígena al colonialismo británico de forma más justa y pertinente que las historias parciales proporcionadas por un grupo de líderes nativos dominantes o historiadores coloniales. Es decir, su posicionamiento epistémico como subalterno cambia estructuralmente el marco epistémico paradigmático creado por las élites occidentales y (post)coloniales en su narración historiográfica. De ahí que la filósofa y teórica literaria hindú Gayatri Chakravorty Spivak (2011) escribiese su ensayo ¿Puede el subalterno hablar?, 
donde, en armonía con Guha, discute la precaria normatividad de esta narración historiográfica. Spivak (2011) afirma que esta precariedad se pone de manifiesto cada vez que el capitalismo occidental establece la universalidad del modo narrativo de producción, y que el hecho de ignorar al individuo subalterno supone continuar con el proyecto imperialista.

El pensamiento crítico postcolonialista de Spivak (2011) apunta que este proceso historiográfico de enfoque eurocéntrico influencia diferentes niveles epistémicos paradigmáticos: global, nacional y local. Poniendo el ejemplo de la abolición del ritual hindú sati (donde las mujeres indígenas se suicidaban en los funerales de sus maridos), por parte del hombre blanco para proteger a la mujer indígena. Spivak (2011) reflexiona críticamente sobre la posibilidad de hablar de los subalternos. Para Spivak (2011), el subalterno oprimido se ve obligado a adoptar costumbres occidentales de conocimiento, pensamiento, razonamiento y lenguaje con el fin de ser oído y atendido. Debido a esta occidentalización, la población subalterna se ve cohibida de reconocer su propia cosmovisión epistemológica y en su lugar debe adoptar una visión epistémica occidental para conocer su mundo no-occidental. En la obra Selected subaltern studies coordinada conjuntamente entre Guha y Spivak (1988), esta última hace una lectura vigotskyana para superar este proceso de "internalización" del sujeto subalterno a través de la mediación cultural dialógica: "el diálogo para Vigotsky es el ejemplo privilegiado de la denominada comunicación de verbalidad directa entre dos autores o fuentes inmediatamente auto-presentes" (p. 24). A través del reconocimiento de la acción dialógica, Spivak (1988) considera que se produce un cruce de líneas que dan acceso a la "micrología" del mundo filosófico de los estudios subalternos. En armonía con esta tendencia que incentiva la creación de identidades propias del mundo subalterno, el científico social hindú especialista en tecnologías Shiv Visvanathan (2003) apela a las heurísticas y a las experiencias de pluralidad, diversidad y complejidad como las bases para nuevos encuentros culturales.

Todas estas perspectivas de intelectuales latinoamericanos, africanos, islámicos e hindúes ponen en evidencia la influencia nociva de la colonialidad, así como la subsecuente imposición de saberes epistémicos eurocéntricos y occidentales. Estas perspectivas epistemológicas del sur nos obligan a pensar el metaparadigma de los ODS desde un marco pluriversal y multirreferencial, en vez de un único modelo monocultural que intente concretizarse como un diseño global. La diversidad epistémica es el único proyecto universal que toda educación libertadora puede diseñar para 
no caer en el "epistemicidio". El reconociendo democrático de otras cosmovisiones epistemológicas provenientes de la población indígena latinoamericana, africana, islámica y asiática deben reconceptualizar la democracia liberal europea/occidental que se viene imponiendo paradigmáticamente y transgredir transcendentalmente su matriz epistémica a través de un pensamiento crítico de frontera capaz de establecer un diálogo horizontal entre todos los pueblos del mundo. Debemos enfocar nuestra mirada en el horizonte paradigmático de los ODS a escala planetaria, buscando construir un mundo donde otros mundos sean posibles, lo que implica reconocer transculturalmente el inmenso jardín que constituye la humanidad. Un jardín en constante proceso evolutivo donde la ciudadanía mundial representa la belleza de una diversidad infinita de flores que deben reconciliar el pasado, el presente y el futuro, para crear una civilización planetaria en armonía medioambiental. Esta polinización transcultural entre las diversas sociedades etnoculturales encuentra en la transdisciplinariedad la herramienta epistemológica para construir puentes espirituales y científicos que interconecten las naciones y los pueblos del mundo, sin privilegiar espacio o tiempo cultural que juzgue o jerarquice la convivencia en el hábitat común de la Tierra. Tal como señala el fundador de la Sociedad Brasileña de Etnomatemáticas, Ubiratan D'Ambrosio (1997): “la transdisciplinariedad reposa sobre una actitud abierta, de respeto mutuo y de humildad con relación a mitos, religiones y sistemas de explicaciones y de conocimiento, rechazando cualquier tipo de arrogancia o prepotencia” (p. 80). Estamos aquí para aconsejarnos mutuamente: plantando semillas de amor y de justicia. De ahí la importancia de las connotaciones filosóficas derivadas de la mecánica cuántica para considerar la entelequia de un espacio-tiempo cultural, religioso, político, artístico o educativo que no pueda jerarquizar los cuadros epistémicos paradigmáticos construidos por el ser humano en su caminar histórico. La transdisciplinariedad representa, entonces, la metodología apropiada para enfrentarnos a la encrucijada paradigmática que representan los ODS.

\section{Últimas reflexiones para abordar la encrucijada paradigmática actual}

El escenario internacional actual de postguerra fría nos incita a pensar que todavía no nos hemos librado de nuestra espada de Damocles contemporánea: la amenaza 
nuclear ${ }^{4}$. La potencialidad nuclear para erradicar cualquier forma de vida en la Tierra ha cambiado paradigmáticamente las normas del juego en las relaciones internacionales, puesto que cualquier grupo terrorista o estado-nación soberano podría destruir el mundo tal y como lo conocemos. Si bien este ultimátum paradigmático que coacciona el desarrollo de la sociedad del riesgo mundial (Beck, 2008) no parece ser un motivo de alarma para crear mecanismos eficaces de gobernabilidad planetaria para gestionar una cultura de paz, la degradación del medio ambiente derivada de nuestros sistemas de producción y consumo ha puesto de manifiesto la insostenibilidad de nuestras prácticas de explotación a la naturaleza: obligándonos a cambiar radicalmente el cuadro epistémico que constituyen nuestras creencias, hábitos, actitudes y valores.

Estamos ante una encrucijada paradigmática que concierne a toda la ciudadanía mundial, sin importar nuestra raza, etnia, cultura, religión o nacionalidad jurídica. La amenaza de un holocausto nuclear y las previsiones de un colapso ecológico (cambio climático, calentamiento global, pérdida de la biodiversidad, etc.) nos compela a desarrollar una profunda transformación en las relaciones del ser humano consigo mismo, con el otro, con la naturaleza y con lo sagrado. De ahí que no podamos excluir ningún tipo de conocimiento o cosmovisión epistémica, bien sea una creencia indígena, una conceptualización artística, una intuición espiritual, una demostración científica o de cualquier otro tipo. La integración e inclusión de todos estos tipos de conocimientos múltiples tiene que ser horizontal y complementaria, puesto que ninguno de ellos podrá nunca albergar un entendimiento completo y definitivo, ya que el conocimiento está abierto a una unidad infinita, como bien demostraron los teoremas de la incompletitud de Gödel. El reconocimiento de la inconmensurabilidad del conocimiento nos obliga a aceptar que cualquier teoría científica, creencia cultural, doctrina política o dogma religioso, serán siempre aproximaciones limitadas a la compleja red de fenómenos que interactúan constantemente en la estructura ontológica de la realidad. Hacer frente a las problemáticas de la globalización requiere una ecología de saberes (Santos, 2010a) cuyo abordaje epistemológico abarque un diálogo transdisciplinar entre las propias disciplinas científicas y los saberes no científicos

4 Como es sabido, entre los años 1958 y 1962, los Estados Unidos y la URSS estuvieron realizando pruebas de explosión nuclear en el espacio exterior. Según estadísticas razonablemente completas, se cree que la URSS y los Estados Unidos (y de manera marginal otros países tecnológicamente avanzados), han lanzado alrededor de 2.200 satélites militares entre 1959 y 1983, duplicándose esa cifra entre los años 1984 y 2000. Si bien se desconocen datos más concluyentes, todo parece indicar que estos satélites militares podrian representar, literalmente, "espadas de Damocles contemporáneas". 
intrínsecos en las culturas ancestrales, la sabiduría indígena, la espiritualidad, las artes y otras formas de organización del conocimiento.

Debemos cuestionarnos nuestro futuro común como especie en una sociedad-mundo enferma endémicamente. Tenemos que tomar consciencia de nuestra patología social y reaccionar en este preciso instante para aliviar los efectos de un cambio climático ya iniciado. Continuando con los mismos modelos de pensamiento, organización socioeconómica insostenible, uso de energías contaminantes, destrucción de los ecosistemas naturales y confrontación bélica, solo estaremos caminando hacia una aceleración de procesos que degradan la naturaleza y nos confinan hacia nuestra propia autodestrucción como especie. Es urgente abandonar los modelos epistémicos paradigmáticos que los postulados capitalistas y el darwinismo social han constituido históricamente desde los siglos XVIII y XIX, puesto que nos han abocado a la crisis civilizatoria y ecológica actual. En este sentido, el sociólogo y analista del sistema-mundo Immanuel Wallerstein (1997) acierta al señalar que "el nuevo tema geocultural ya ha sido proclamado: es el tema de la identidad; identidad en cuanto incrustada en un concepto huidizo llamado cultura o, para ser más exacto, culturas" (p. 91). En efecto, debemos promover nuevos acercamientos epistemológicos para concebir que nuestra identidad es construida a partir de múltiples relaciones naturales y sociales, entendiendo que toda cultura es más o menos híbrida, mestiza, hecha de cruces, retroalimentaciones... No existen culturas perfectas ni acabadas. Toda cultura lleva en sí misma suficiencias, insuficiencias, funcionalidades, disfuncionalidades... Como señala Dussel (2006) "las “eticidades" de la humanidad se fueron generando en torno y desde un sistema asiático-africano-mediterráneo, que desde el siglo XV es, por primera vez, un sistema mundial" (p. 20). De ahí que debamos promover un diálogo intercultural sin jerarquizaciones que desarrolle y potencialice alternativas epistémicas de cada cultura para conseguir los ODS, puesto que solo la fecundación transcultural entre los pueblos nos podrá salvar de la barbarie a la que estamos encaminados como sistema-mundo. 


\section{Referencias}

Beck, U. (2008). La sociedad del riesgo mundial. En busca de la seguridad perdida. Barcelona: Paidós.

Castells, M. (2000). La era de la información. La sociedad red, 1(2). Torrejón de Ardoz: Alianza Editorial.

Deleuze, G., y Guattari, F. (2006). Mil mesetas: capitalismo y esquizofrenia. Valencia: Pre- Textos.

Dussel, E. (2006). Ética de la liberación en la edad de la globalización y de la exclusión.

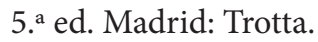

Feyerabend, P. K. (1997). Tratado contra el método: esquema de una teoría anarquista del conocimiento. 3. ${ }^{\text {e }}$. Madrid: Tecnos.

Goody, J. (2006). The theft of history. Cambridge: Cambridge University Press.

Grosfoguel, R. (2006). La descolonización de la economía política y los estudios postcoloniales: transmodernidad, pensamiento fronterizo y colonialidad global. En Tabula Rasa, (4), 17-48. Bogotá.

Guha, R. (Ed.). (1982). Subaltern studies I: Writing on South Asian history and society. New Delhi: Oxford University Press.

Guha, R., y Chakravorty S, G. (ed.). (1988). Selected subaltern studies. New York: Oxford University Press.

Hountondji, P. J. (2010). Conhecimento de África, conhecimentos africanos: duas perspectivas sobre os estudos africanos. En Santos B. S., y Meneses, M. P. (Eds.). Epistemologias do Sul (pp. 131-144). São Paulo: Cortez.

Klein, J. T. (1994). Notes toward a social epistemology of transdisciplinarity. Comunicación presenta en el I Congreso Mundial de la Transdisciplinaridad.

Kuhn, T. (1970). The structure of scientific revolutions. 2. ${ }^{\text {a }}$ ed. Chicago: The University of Chicago.

Masolo, D. A. (2010). Filosofia e conhecimento indígena: uma perspectiva africana. En Boaventura, S. S., y Meneses, M. P. (Eds.). Epistemologias do Sul (pp. 313-240). São Paulo: Cortez. 
Mignolo, W. (2003). Historias locales/diseños globales: colonialidad, conocimientos subalternos y pensamiento fronterizo. Madrid: Akal.

Mignolo, W. (2001). Capitalismo y geopolítica del conocimiento. El eurocentrismo y la filosofía de la liberación en el debate intelectual contemporáneo. Buenos Aires: El Signo.

Moosa, E. (2010). Transições no "progresso” da civilização: teorização sobre a história, a prática e a tradição. En Boaventura, S. S., y Meneses, M. P. (Eds.). Epistemologias do Sul (pp. 291-312). São Paulo: Cortez.

Morin, E. (2008). Introdução ao pensamento complexo. Lisboa: Instituto Piaget.

Nicolescu, B. (2008). O manifesto da transdisciplinaridade. São Paulo: TRIOM.

Quijano, A. (2001). Colonialidad del poder. Cultura y conocimiento en América Latina. En Walter Mignolo (Ed.). Capitalismo y geopolítica del conocimiento. El eurocentrismo y la filosofía de la liberación en el debate intellectual contemporáneo (pp. 117-132). Buenos Aires: El signo.

Quijano, A. (2010). Colonialidade do poder e classificação social. En Boaventura S. S., y Meneses, M. P. (Eds.). Epistemologias do Sul (pp. 84-130). São Paulo: Cortez.

Ramose, M. B. (2010). Globalização e ubuntu. En Boaventura S. S., y Meneses, M. P. (Eds.). Epistemologias do Sul (pp. 175-220). São Paulo: Cortez.

Riechmann, J. (2014). Un buen encaje en los ecosistemas. 2. ${ }^{\text {a }}$ ed. (revisada) de Biomímesis. Madrid: Ed. Catarata.

Roland, R. (1992). Globalization. Social theory and global culture. London: SAGE.

Santos, B. S. (1995). Toward a new common sense: Law, sciencie, and politics in the paradigmatic transition. New York: Routledge.

Santos, B. S. (2010a). Para além do pensamento abyssal: das linhas globais a uma ecologia de saberes. En Boaventura S. S., y Meneses, M. P. (Eds.). Epistemologias do Sul (pp. 31-83). São Paulo: Cortez.

Santos, B. S. (2010b). Um ocidente não-ocidentalista? A filosofia à venda, a douta ignorância e a aposta de Pacal. En Boaventura S. S., y Meneses, M. P. (Eds.). Epistemologias do Sul (pp. 519-562). São Paulo: Cortez. 
Spivak, G. C. (2011). ¿Puede hablar el subalterno? Buenos Aires: El cuenco de plata. Visvanathan, S. (2003). Cultural encounters and the orient: A study in the politics of knowledge. En Diogenes, 50(4), 69-81.

Wallerstein, I. (1997). El futuro de la civilización capitalista. Barcelona: Icaria. 